\title{
An Index for Measuring Functional Diversity in Plant Communities Based on Neural Network Theory
}

\author{
Naiqi Song ${ }^{1}$ and Jin-Tun Zhang $^{2}$ \\ ${ }^{1}$ School of Mathematical Sciences, Beijing Normal University, Beijing 100875, China \\ ${ }^{2}$ College of Life Sciences, Beijing Normal University, Beijing 100875, China \\ Correspondence should be addressed to Jin-Tun Zhang; zhangjt@bnu.edu.cn
}

Received 26 February 2013; Revised 8 May 2013; Accepted 14 May 2013

Academic Editor: Hector Pomares

Copyright (c) 2013 N. Song and J.-T. Zhang. This is an open access article distributed under the Creative Commons Attribution License, which permits unrestricted use, distribution, and reproduction in any medium, provided the original work is properly cited.

Functional diversity in plant communities is a key driver of ecosystem processes. The effective methods for measuring functional diversity are important in ecological studies. A new method based on neural network, self-organizing feature map (SOFM index), was put forward and described. A case application to the study of functional diversity of Phellodendron amurense communities in Xiaolongmen Forest Park of Beijing was carried out in this paper. The results showed that SOFM index was an effective method in the evaluation of functional diversity and its change in plant communities. Significant nonlinear correlations of SOFM index with the common used methods, FAD, MFAD, FDp, FDc, FRic, and FDiv indices, also proved that SOFM index is useful in the studies of functional diversity.

\section{Introduction}

Functional diversity in plant communities is a key driver of ecosystem processes, ecosystem resilience to environmental change, and ecosystem services [1-3]. Functional diversity refers to the value and scope of species functional traits in a special ecosystem, also called functional trait diversity (FTD). It stressed differences of functions among species in communities $[4,5]$. The differences in types, change amplitude, and stability of functional traits reflect the quantity of functional diversity in community $[2,5]$. The effective methods for measuring functional diversity in plant community are essential in the studies of functional diversity [6].

In the last two decades, a growing number of methods for measuring functional diversity have been proposed and applied. These methods differ in mathematical properties, the features they capture, their emphasis on location or dispersion measures, the consideration of single or multiple traits, and the inclusion of abundances of the trait values. Walker et al. [7] proposed a functional diversity index by identifying the number of different combinations of trait values. Most of the later approaches were based on dissimilarity among species in trait space. Functional attribute diversity is the sum of all dissimilarities among pairs of species using ecological distance [7]. Petchey and Gaston [5] proposed the use of a dendrogram to obtain functional diversity indices using multitrait profiles without abundance. Casanoves et al. [8] developed the software (FDiversity) for these methods. Zhang et al. [2] introduce fuzzy equivalence relation to measure functional diversity of plant community. Although some applications and comparisons have proved these methods to be useful in functional diversity analysis $[2,8-10]$, the effective methods for measuring functional diversity are still a limit in practical studies $[3,8]$.

Artificial neural networks (ANNs) have already been successfully used in ecology [11-13]. Based on the mechanism of the human brain, the Artificial Neural Network is a convenient alternative tool to traditional statistical methods. The Kohonen Self-Organizing Feature Map (SOFM) is one of the most well-known neural networks with unsupervised learning rules [11, 14-16], and it performs a topology-preserving projection of the data space onto a regular two-dimensional space. It has been successfully used in classification and ordination of plant communities $[16,17]$. Because of its theoretical advantages, it is expected to apply Self-Organizing 


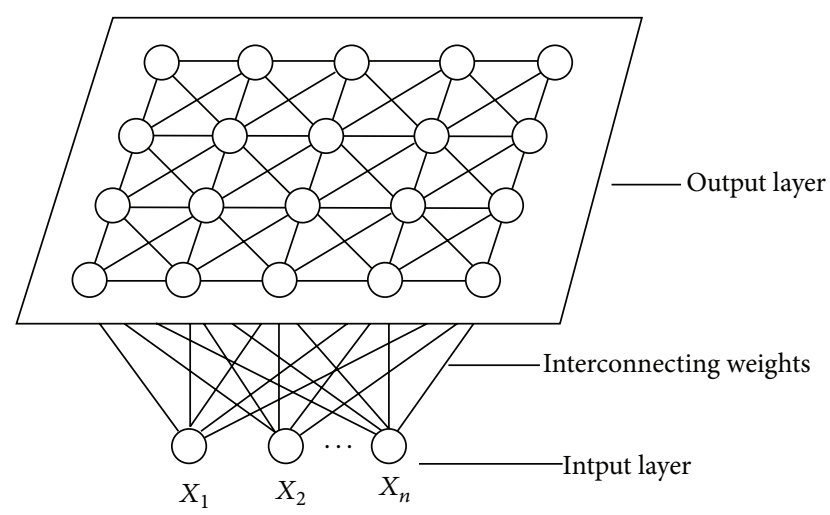

FIGURE 1: The structure of self-organizing feature map (SOFM) neural network.

Feature Map to functional diversity analysis. In this paper, the SOFM algorithm was applied to quantify functional diversity of plant communities. Its results were compared and tested with several present effective methods in plant ecology.

\section{Methods}

2.1. Self-Organizing Feature Map (SOFM). SOFM neural network uses unsupervised learning and produces a topologically ordered output that displays the similarity between the species presented to it $[18,19]$. The network consists of two layers, input layer and output layer (Figure 1). The input layer contains a unit (neuron) for each variable (species functional trait) in the dataset. The input units operate in a similar way to those in other neural networks, effectively presenting the data for each species to the network in an appropriate format. The input units are connected directly to units in the output layer or competitive layer. The output layer is also a two-dimensional array of units, and each of these units is connected to every unit in the input layer by a weighted connection. Lateral interaction between units in the output layer also ensures that learning is a competitive process in which the network adapts to respond in different locations for inputs that differ. Consequently, species that are similar should be associated with units that are close together in the output layer while a dissimilar species would be associated with a distant unit elsewhere in the output layer. The projection depicted by the SOFM output is non-linear $[15,19]$.

2.2. SOFM Index for Measuring Functional Diversity. Suppose that the input data vector is

$$
P_{k}=\left(P_{1}^{k}, P_{2}^{k}, \ldots, P_{N}^{k}\right), \quad(k=1,2, \ldots, q) .
$$

Suppose that the associated weight vector is

$$
\begin{array}{r}
W_{i j}=\left(W_{j 1}, W_{j 2}, \ldots, W_{j i}, \ldots, W_{j N}\right), \\
i=1,2, \ldots, N ; j=1,2, \ldots, M .
\end{array}
$$

Then, the procedure of SOFM functional diversity measure is presented as follows.
(1) Initializing. Giving initial values of $W_{i j}$ within $[0,1]$ randomly $(i=1,2, \ldots, N ; j=1,2, \ldots, M)$, initial values of learning rate $\eta(0)$ and neighborhood $N_{g}(0)$, as well as determining total learning times $T$.

(2) Inputting a random species unit drawn from the input dataset $P_{k}$ into the network and calculating $\bar{P}_{k}$ as follows:

$$
\bar{P}_{k}=\frac{P_{k}}{\left\|P_{k}\right\|}=\frac{\left(P_{1}^{k}, P_{2}^{k}, \ldots, P_{N}^{k}\right)}{\left[\left(P_{1}^{k}\right)^{2}+\left(P_{2}^{k}\right)^{2}+\cdots+\left(P_{N}^{k}\right)^{2}\right]^{1 / 2}} .
$$

(3) Calculating $\bar{w}_{j}$ as follows:

$$
\bar{W}_{j}=\frac{W_{j}}{\left\|W_{j}\right\|}=\frac{\left(W_{j 1}, W_{j 2}, \ldots, W_{j N}\right)}{\left[\left(W_{j 1}\right)^{2}+\left(W_{j 2}\right)^{2}+\cdots+\left(W_{j N}\right)^{2}\right]^{1 / 2}} .
$$

(4) Defining Euclidean distance between $\bar{w}_{j}$ and $\bar{P}_{k}$ as follows:

$$
d_{j}=\left[\sum_{i=1}^{N}\left(\overline{P_{i}^{k}}-\overline{W_{j i}}\right)^{2}\right]^{1 / 2}, \quad(j=1,2, \ldots, M) .
$$

(5) Determining the minimum distance $d_{g}$, with $g$ chosen as the winning neuron and called the Best Matching Unit (BMU), as follows:

$$
d_{g}=\min \left[d_{j}\right], \quad j=1,2, \ldots, M .
$$

(6) Adjusting the weights $\left(W_{i j}\right)$ as follows:

$$
\begin{array}{r}
\overline{W_{j i}(t+1)}=\overline{W_{j i}(t)}+\eta(t) \cdot\left[\overline{P_{i}^{k}}-\overline{W_{j t}(t)}\right], \\
(j=1,2, \ldots, M),
\end{array}
$$

where $\eta(t)$ is the learning rate at $t$ time being defined as follows:

$$
\eta(t)=\eta(0)\left(1-\frac{t}{T}\right) \quad(0<\eta(0)<1) .
$$

The neighborhood $N_{g}(t)$ is defined as

$$
N_{g}(t)=\operatorname{INT}\left[N_{g}(0)\left(1-\frac{t}{T}\right)\right],
$$

where $N_{g}(0)$ is initial value of $N_{g}(t)$.

(7) Increasing time $t$ to $t+1$. If $t<T$, then go to step (2); if else, stop the training.

When the learning process is finished, a topological map of small squares is obtained, and species units can be mapped into the corresponding squares. This map reflects the distribution of species in functional trait space.

(8) Scaling the two-dimensional axes: considering the topological map of small squares of $6 \times 6$ a diagram with coordinate scores of $0.0-0.6$, with each species having its twodimensional scores.

(9) Calculating functional distances between species as follows:

$$
d_{j k}=\sqrt{\left(x_{j}-x_{k}\right)^{2}+\left(y_{j}-y_{k}\right)^{2}} \text {, }
$$


where $d_{j k}$ is functional distance between species $j$ and $k$ in topological space, $x_{j}$ and $x_{k}$ are scores of species $j$ and $k$ on $x$-axis, and $y_{j}$ and $y_{k}$ are scores of species $j$ and $k$ on $y$-axis.

(10) Calculating SOFM functional diversity as

$$
\begin{aligned}
& \operatorname{SOFM}_{\mathrm{FD}}=\frac{1}{2} \sum d_{j k} \\
& (j=k=1,2, \ldots, \text { and } S=\text { species number in a quadrat }) .
\end{aligned}
$$

The parameterization of the SOFM used was that the learning rate was 0.1 for the ordinating phase and 0.02 for adjusting phase; the learning phase was broken down into 5000 steps for the ordinating phase and 50000 steps for the tuning phase.

2.3. Compared Methods. To test the effects of SOFM index, six common methods were applied, and their results were compared with that of SOFM method.

2.3.1. Functional Attribute Diversity (FAD). FAD aimed at estimating the dispersion of species in trait space as the sum of the pairwise species distances [7]:

$$
\mathrm{FAD}=\sum_{i, j} d_{i j}
$$

where $d_{i j}$ is functional distance between species $i$ and $j$ in functional trait space; $D=\left\{d_{i j}\right\}$ is Euclidean distance matrix and calculated based on the matrix of functional traits $(N)$ by species $(S)$.

2.3.2. Modified FAD (MFAD). For a given set of data of $S$ species and $N$ traits, the functional species were defined. The set of functional species results from combining the species with the same values in all of the traits into only one functional species. The number of entities in the data matrix will be reduced from $S$ to $M(M \leq S)$, and accordingly the pairwise dissimilarities are reduced from an $S \times S$ to an $M \times M$ matrix. MFAD is calculated as

$$
\text { MFAD }=\frac{\sum_{i, j}^{M} d_{i j}}{M},
$$

where $d_{i j}$ is the distance between functional units " $i$ " and " $j$ ", and $M$ is the number of functional units.

2.3.3. Functional Diversity Based on Dendrogram. Functional diversity index based on dendrogram is the total branch length of the functional dendrogram that can be constructed from information about species functional traits [10]. There were the two following choices. First, FDp is plot-based index which recalculates the dendrogram for each quadrat, but in doing so the desirable property of "set monotonicity" does not hold. Second, FDc is community-based index which corrects the lack of monotonicity that arises when there is a particular dendrogram for each quadrat.

2.3.4. Functional Richness (FRic). FRic represents the trait space filled by the community. With more than one trait, it is represented by the volume filled by the community in the trait space. The procedure is like the convex hull hypervolume [20]. The algorithm identifies the extreme species and then estimates the volume in the trait space. The maximum value of FRic in a $T$ dimensional trait space is attained with $2^{T}$ species with a combination of extreme values of the traits values.

2.3.5. Functional Divergence (FDiv). Functional divergence is related to how abundance is distributed within the volume of functional traits space. The functional divergence index is

$$
\mathrm{FDiv}=\frac{\sum_{i=1}^{S} w_{i}\left(d G_{i}-\overline{d G}\right)+\overline{d G}}{\sum_{i=1}^{S} w_{i}\left|d G_{i}-\overline{d G}\right|+\overline{d G}},
$$

where $d G_{i}$ is the functional distance from species $i$ to the gravity center of species that form the vertices of the convex hull, $\overline{d G}$ is the mean distance of the $S$ species to the gravity center, $w_{i}$ is the relative abundance of species $i$.

\section{Case Study}

3.1. Vegetation Data. The new SOFM method was applied and compared in functional analysis of Phellodendron amurense communities in Xiaolongmen Forest Park of Beijing, China. Twenty-five sampling quadrats of Phellodendron amurense communities were set up. Species data were recorded in each quadrat. The quadrat size was $10 \mathrm{~m} \times 20 \mathrm{~m}$, in which three $5 \mathrm{~m} \times 5 \mathrm{~m}$ and three $2 \mathrm{~m} \times 2 \mathrm{~m}$ small quadrats were used to record shrubs and herbs, respectively. Species name, cover, height, basal area, and individual abundance for tree species as well as cover, abundance, and height for shrubs and herbs were recorded in each quadrat. The plant height was measured by using a height meter for trees and a ruler for shrubs and herbs. The basal diameter of trees was measured by using a caliper and was used to calculate the basal area. A total of 105 plant species were recorded in 25 quadrats [21].

Nine functional traits were selected to illustrate plant species functions in community (Table 1). Photosynthesis pathway, seed dispersal, pollination manner, and nitrogen fixing were identified from local flora while life form, leaf form, plant height, flowering date, and flowering period were observed and measured in situ. To calculate functional diversity, a data matrix of functional traits $\times$ species in a quadrat was constructed, and there were in total 25 data matrices for 25 quadrats. All functional trait data were standardized before merging the species trait information to the species in each quadrat and calculating functional diversity to avoid scale effects [8].

\section{Results}

According to SOFM procedure, we calculated SOFMFD index for each quadrat (Table 2). These indices successfully described the functional diversity in communities. The change of functional diversity of Phellodendron amurense communities is great, that is, from 40.7 to 209.5. This change may be dependent on interaction with environmental 
TABLE 1: Plant functional traits and their values in plant communities.

\begin{tabular}{ll}
\hline Functional trait type & Functional traits and values \\
\hline Photosynthesis pathway & 1 Crassulacean pathway, 2 C3 pathway, 3 between C3 and C4 pathway, and 4 C4 pathway \\
Nitrogen fixing & 0 No nitrogen fixing, 1 Elaeagnaceae nitrogen fixing, and 2 Leguminosae nitrogen fixing \\
Seed dispersal & 1 Automatic spreading, 2 gravity spreading, 3 wind spreading, and 4 animals spreading \\
Pollination method & 1 Anemophilous, 2 entomophilous \\
Life form & 1 Tree, 2 shrubs, 3 woody vine, 4 perennial herb, 5 biennial herb, and 6 annual herb \\
Leaf form & 1 Coniferous, 2 Broad leaf \\
Plant height & Measured value in cm \\
Flowering date & Beginning month of flowering \\
Flowering period & Flowering months \\
\hline
\end{tabular}

TABLE 2: SOFM functional diversity values for 25 quadrats of Phellodendron amurense communities.

\begin{tabular}{lccc}
\hline Quadrat & SOFMFD & Quadrat & SOFMFD \\
\hline 1 & 58.5 & 14 & 124.3 \\
2 & 97.9 & 15 & 90.8 \\
3 & 88.3 & 16 & 198.5 \\
4 & 58.9 & 17 & 187.4 \\
5 & 53.9 & 18 & 190.0 \\
6 & 83.4 & 19 & 200.2 \\
7 & 85.7 & 20 & 189.3 \\
8 & 99.2 & 21 & 194.2 \\
9 & 62.9 & 22 & 193.3 \\
10 & 123.0 & 23 & 57.3 \\
11 & 59.9 & 24 & 40.7 \\
12 & 130.0 & 25 & 42.2 \\
13 & 209.5 & & \\
\hline
\end{tabular}

gradient. This could be proved by the relationships between SOFMFD functional diversity and elevation (Figure 2), which suggested that SOFMFD also successfully described the variation of functional diversity along environmental gradient in Phellodendron amurense communities.

SOFMFD index was significantly correlated with the six methods, FAD, MFAD, FDc, FDp, FRic, and FDiv indices (Figure 3). Their relations were all non-linear; that is, the relations of SOFMFD index with FAD, MFAD, FDc, FDp, and FRic indices were all sigmoid curves (best fitted), and the relation of SOFMFD index with FDiv index was an exponential curve (best fitted) (Figure 3). These six methods are common and comparatively widely used in functional diversity studies. Their significant non-linear relations with SOFMFD index further proved that SOFMFD index is an effective method in functional diversity analysis in plant communities.

\section{Discussion}

SOFMFD index successfully described the quantity and change of functional diversities of Phellodendron amurense communities which suggested that SOFMFD index can

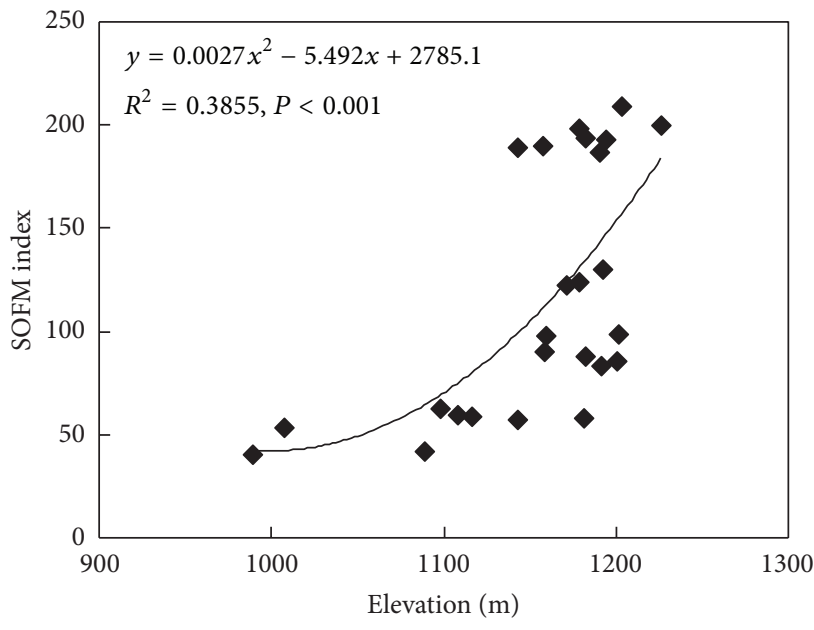

FIGURE 2: Relationship between SOFM functional diversity and elevation.

perfectly complete the evaluation of functional diversity and is fully usable in plant ecology. Although there are no generalized standards to evaluate a new method, it is an effective technique if a method can be used in describing ecological relations and can provide reasonable results [22, 23].

Significant correlations of SOFMFD index with common used methods, FAD, MFAD, FDc, FDp, FRic, and FDiv indices, also proved that SOFMFD index is an effective method to quantify functional diversity in plant communities $[10,20]$. Their relations were all non-linear which suggested that the SOFM index could provide different information than others [8]. The six indices used in this study, FAD, MFAD, FDc, FDp, FRic, and FDiv, are all distance-based methods. They were measured in functional trait space, and the distances between species were calculated by use of functional traits $\times$ species matrix directly [24]. While SOFM index was measured in neural network topological space of functional traits, it is theoretically different from other indices, it can deal with much imprecise and incomplete fuzzy information, and it has advantages in solving non-linear 


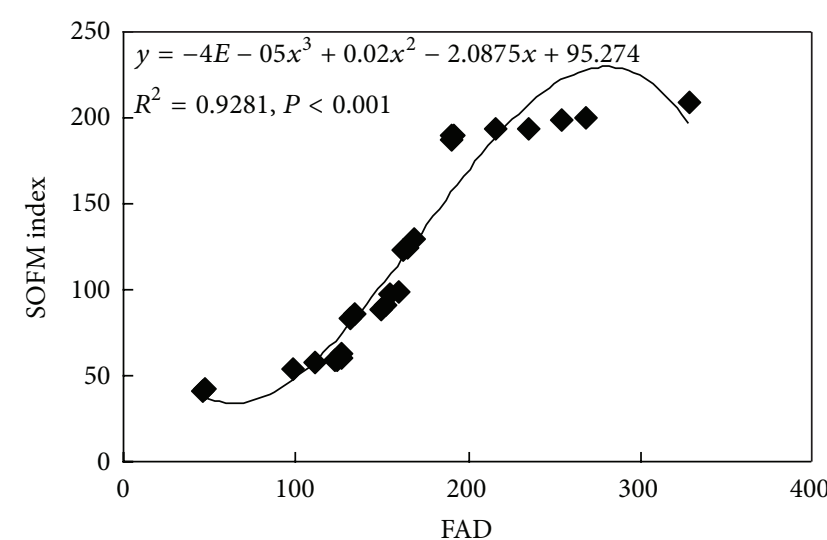

(a)

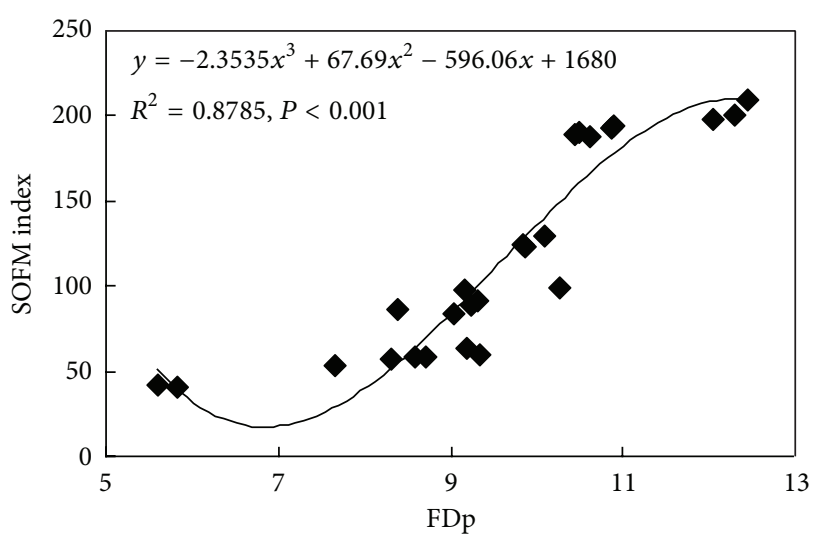

(c)

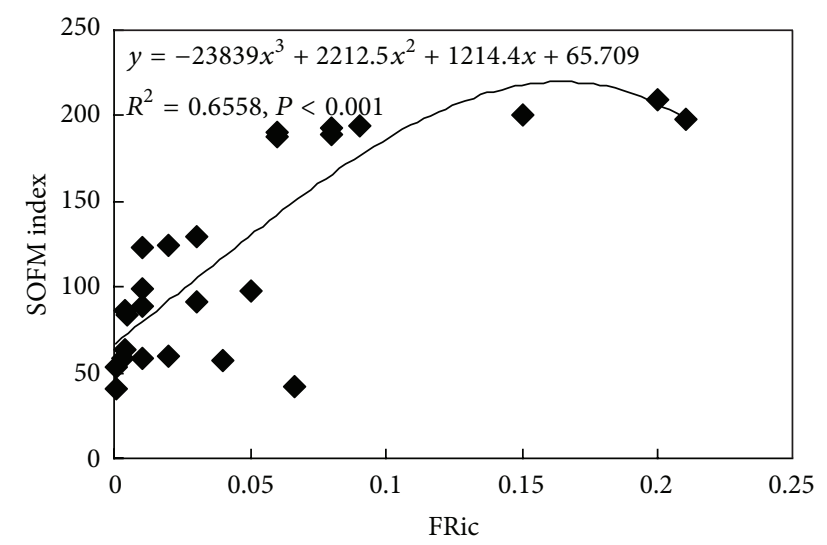

(e)

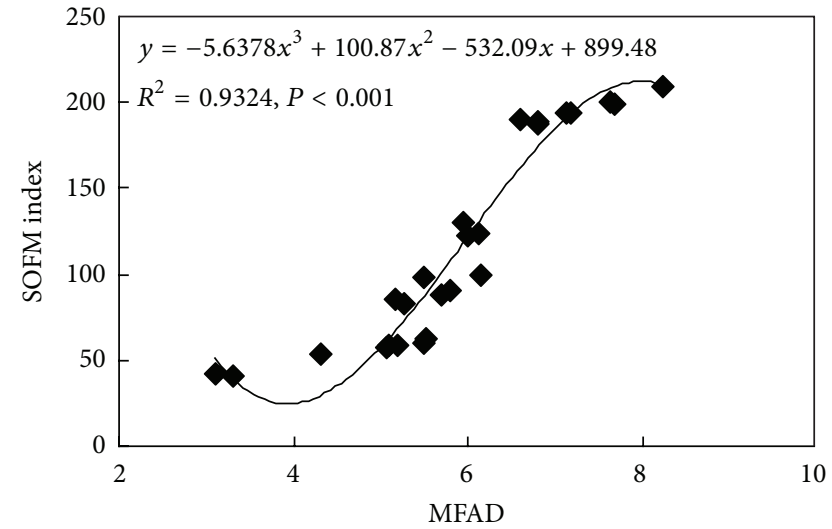

(b)

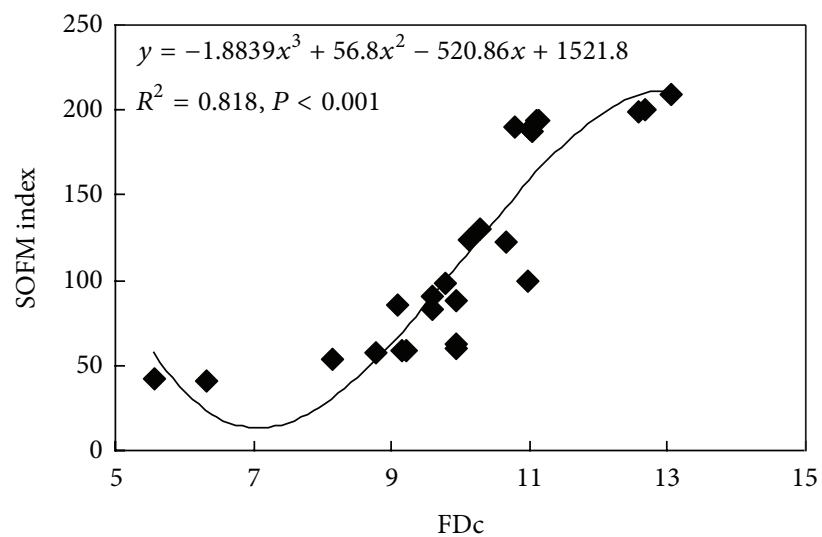

(d)

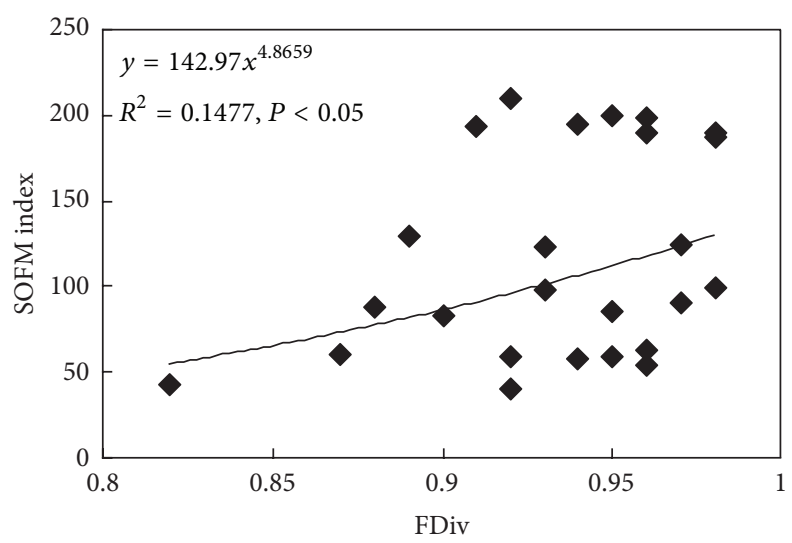

(f)

FIGURE 3: Regression analyses of SOFMFD with FAD, MFAD, FDp, FDc, FRic, and FDiv indices.

problems and in studying complicated systems [24]. Theoretically, SOFM can describe natural phenomena and rules better $[2,17]$. The network can distribute work parallel and hence can calculate very quickly. It can distribute information within the whole network with variation of weights, and problems for some units cannot affect the network function. Therefore, it is very suitable for analyzing complex systems $[20,23]$. Vegetation ecosystem is such a complexity system with various non-linear and fuzzy relations of species, communities, and environmental factors. Therefore, SOFMFD index for measuring functional diversity should be perfect technique in the study of plant community [8].

As a new method, SOFM index needs more applications and case studies in different types of plant communities in different climatic regions to test its validity and advantages in future $[2,24]$. 


\section{Acknowledgments}

This study was financially supported by the Specialized Research Fund for the Doctoral Program of Higher Education (Grant no. 20120003110024) and the National Natural Science Foundation of China (Grant no. 31170494). The authors thank the editor and reviewers for their constructive comments and suggestions on this paper.

\section{References}

[1] S. Díaz, S. Lavorel, F. de Bello, F. Quétier, K. Grigulis, and T. M. Robson, "Incorporating plant functional diversity effects in ecosystem service assessments," Proceedings of the National Academy of Sciences of the United States of America, vol. 104, no. 52, pp. 20684-20689, 2007.

[2] J. T. Zhang, L. H. Fan, and M. Li, "Functional diversity in plant communities: theory and analysis methods," African Journal of Biotechnology, vol. 11, pp. 1014-1022, 2012.

[3] F. de Bello, "The quest for trait convergence and divergence in community assembly: are null-models the magic wand?" Global Ecology and Biogeography, vol. 21, pp. 312-317, 2012.

[4] D. Tilman, P. B. Reich, J. Knops, D. Wedin, T. Mielke, and C. Lehman, "Diversity and productivity in a long-term grassland experiment," Science, vol. 294, no. 5543, pp. 843-845, 2001.

[5] O. L. Petchey and K. J. Gaston, "Functional diversity (FD), species richness and community composition," Ecology Letters, vol. 5, no. 3, pp. 402-411, 2002.

[6] S. Villéger, N. W. H. Mason, and D. Mouillot, "New multidimensional functional diversity indices for a multifaceted framework in functional ecology," Ecology, vol. 89, no. 8, pp. 2290-2301, 2008.

[7] B. Walker, A. Kinzig, and J. Langridge, "Plant attribute diversity, resilience, and ecosystem function: the nature and significance of dominant and minor species," Ecosystems, vol. 2, no. 2, pp. 95-113, 1999.

[8] F. Casanoves, L. Pla, J. Di Rienzo, and S. Diaz, "FDiversity: a software package for the integrated analysis of functional diversity," Methods in Ecology and Evolution, vol. 2, no. 3, pp. 233-237, 2011.

[9] N. W. H. Mason, D. Mouillot, W. G. Lee, and J. B. Wilson, "Functional richness, functional evenness and functional divergence: the primary components of functional diversity," Oikos, vol. 111, no. 1, pp. 112-118, 2005.

[10] O. L. Petchey and K. J. Gaston, "Functional diversity: back to basics and looking forward," Ecology Letters, vol. 9, no. 6, pp. 741-758, 2006.

[11] T. S. Chon, Y. S. Park, K. H. Moon, and E. Y. Cha, "Patternizing communities by using an artificial neural network," Ecological Modelling, vol. 90, no. 1, pp. 69-78, 1996.

[12] S. Lek and J. F. Guegan, "Artificial Neuronal Networks application to ecology and evolution," in Environmental Science, S. Lek and J. F. Guegan, Eds., Springer, Berlin, Germany, 2000.

[13] J. T. Zhang, N. Q. Song, and M. Li, "Application of fuzzy equivalence clustering to the analysis of functional diversity in plant communities," in Proceedings of the 9th International Conference on Fuzzy Systems and Knowledge Discovery, vol. 2, pp. 556-560, IEEE \& CAS, 2012.

[14] J. L. Giraudel and S. Lek, "A comparison of self-organizing map algorithm and some conventional statistical methods for ecological community ordination," Ecological Modelling, vol. 146, no. 1-3, pp. 329-339, 2001.

[15] R. K. Peet and O. L. Loucks, "A gradient analysis of southern Wisconsin forests," Ecology, vol. 58, pp. 485-499, 1977.

[16] J. T. Zhang and M. Li, "Application of self-organizing feature map clustering and ordination to the analysis of subalpine meadows in North China," in Proceedings of the 6th International Conference on Natural Computation (ICNC'10), vol. 3, pp. 1564-1568, IEEE CAS, August 2010.

[17] J. Zhang, S. Li, and M. Li, "A comparison of self-organizing feature map clustering with TWINSPAN and fuzzy C-means clustering in the analysis of woodland communities in the Guancen Mts, China," Community Ecology, vol. 11, no. 1, pp. 120126, 2010.

[18] R. J. Schalkoff, Pattern Recognition: Statistical, Structural and Neural Approaches, John Wiley \& Sons, New York, NY, USA, 1992.

[19] G. M. Foody, "Applications of the self-organising feature map neural network in community data analysis," Ecological Modelling, vol. 120, no. 2-3, pp. 97-107, 1999.

[20] K. N. Suding, S. Lavorel, F. S. Chapin III et al., "Scaling environmental change through the community-level: a trait-based response-and-effect framework for plants," Global Change Biology, vol. 14, no. 5, pp. 1125-1140, 2008.

[21] Y. Z. Huo, Beijing Physical Geography, Beijing Normal University Press, Beijing, China, 1989.

[22] D. Mouillot, W. H. N. Mason, O. Dumay, and J. B. Wilson, "Functional regularity: a neglected aspect of functional diversity," Oecologia, vol. 142, no. 3, pp. 353-359, 2005.

[23] J. T. Zhang, Quantitative Ecology, Science Press, Beijing, China, 2nd edition, 2011.

[24] J. T. Zhang, Y. Dong, and Y. Xi, "A comparison of SOFM ordination with DCA and PCA in gradient analysis of plant communities in the midst of Taihang Mountains, China," Ecological Informatics, vol. 3, no. 6, pp. 367-374, 2008. 


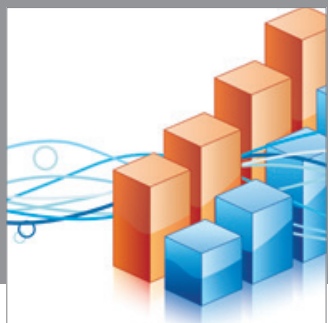

Advances in

Operations Research

mansans

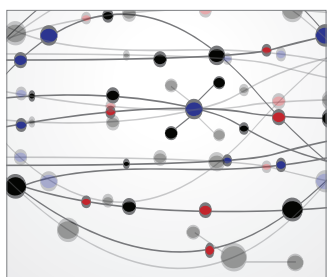

The Scientific World Journal
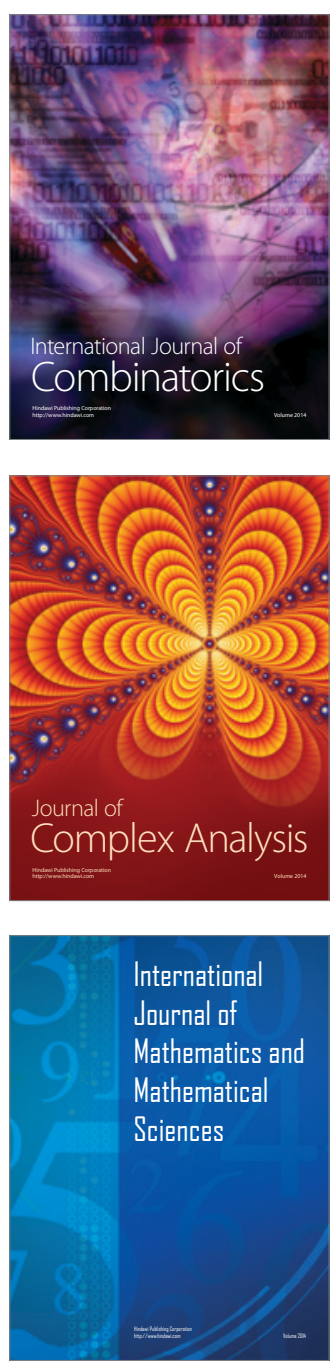
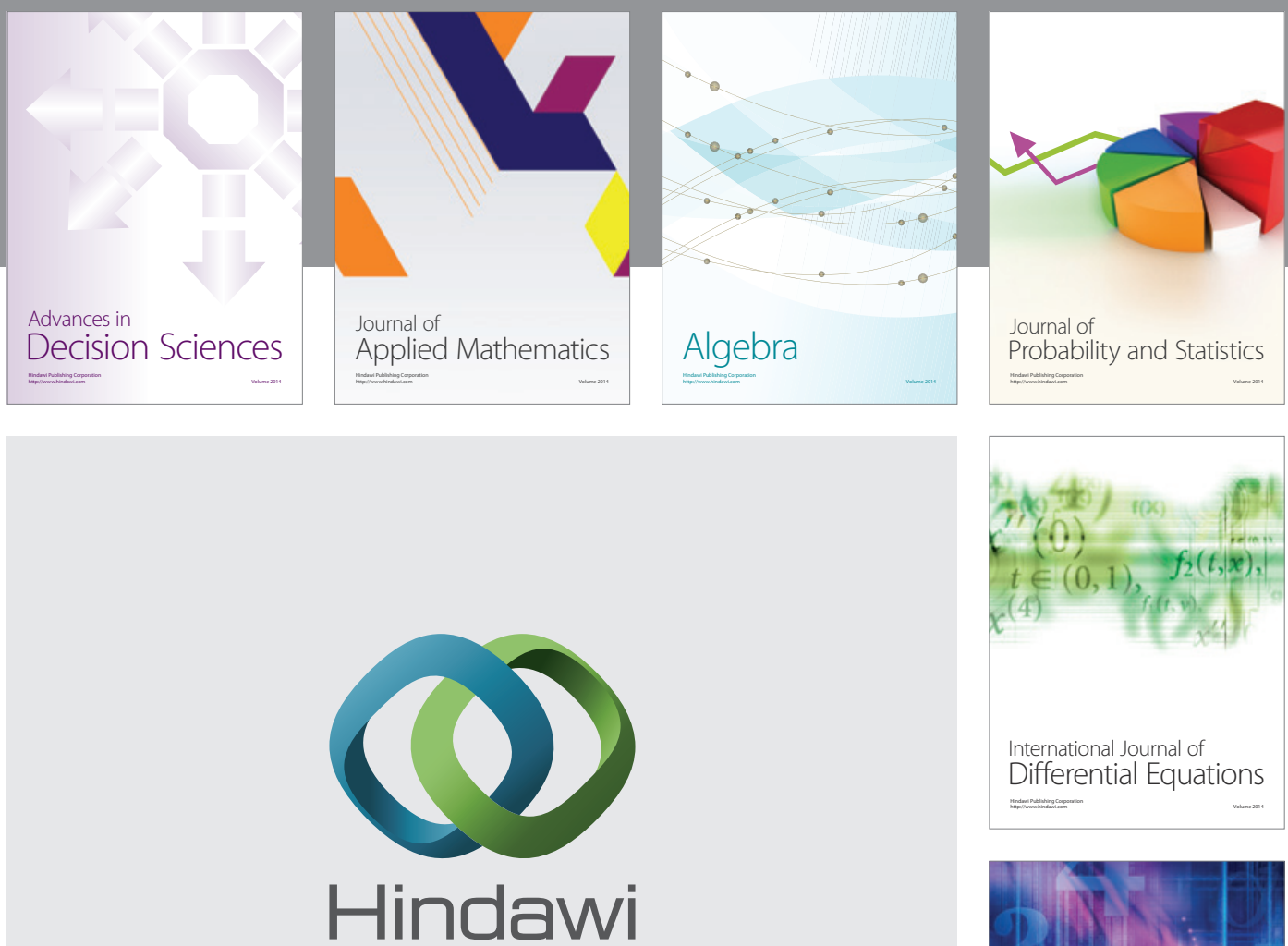

Submit your manuscripts at http://www.hindawi.com
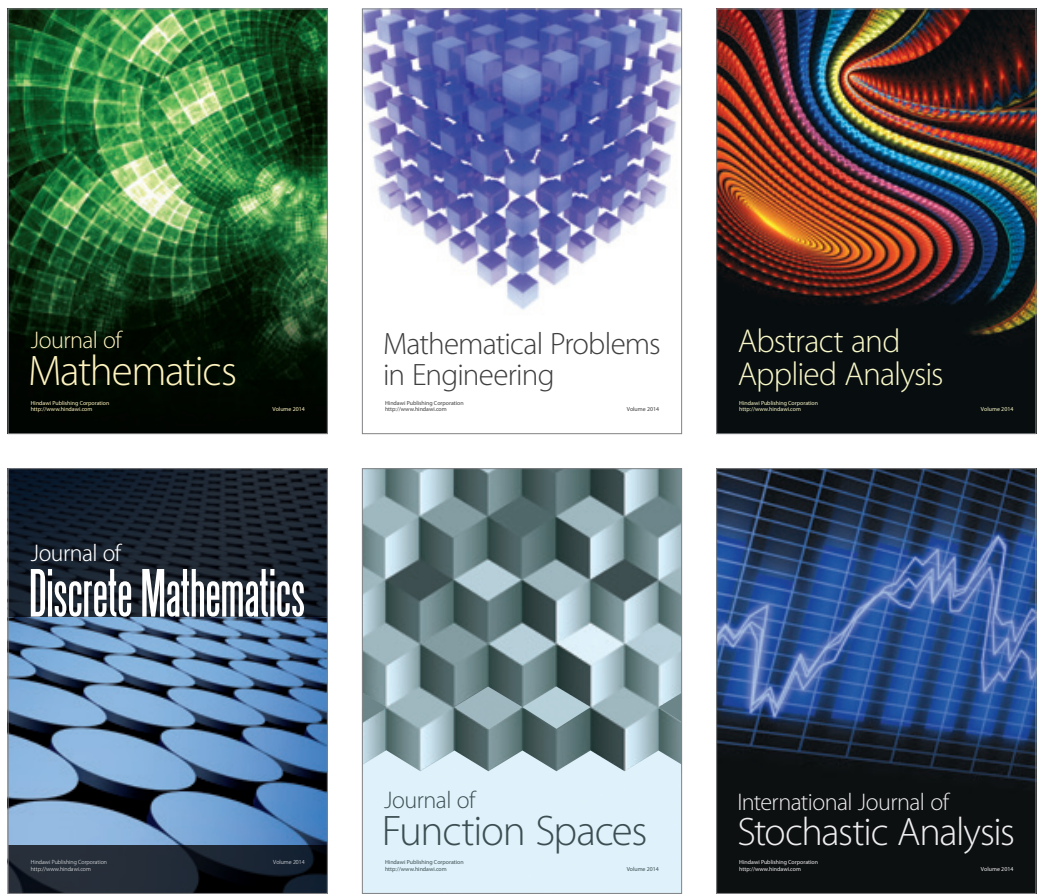

Journal of

Function Spaces

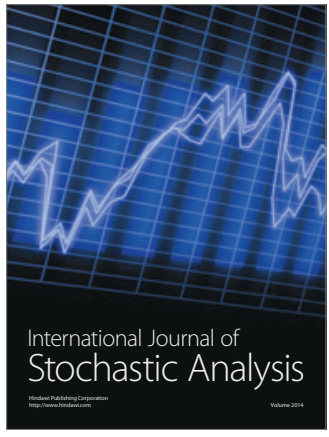

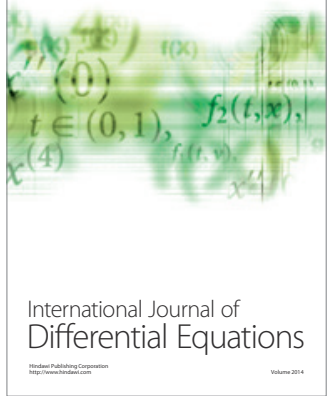
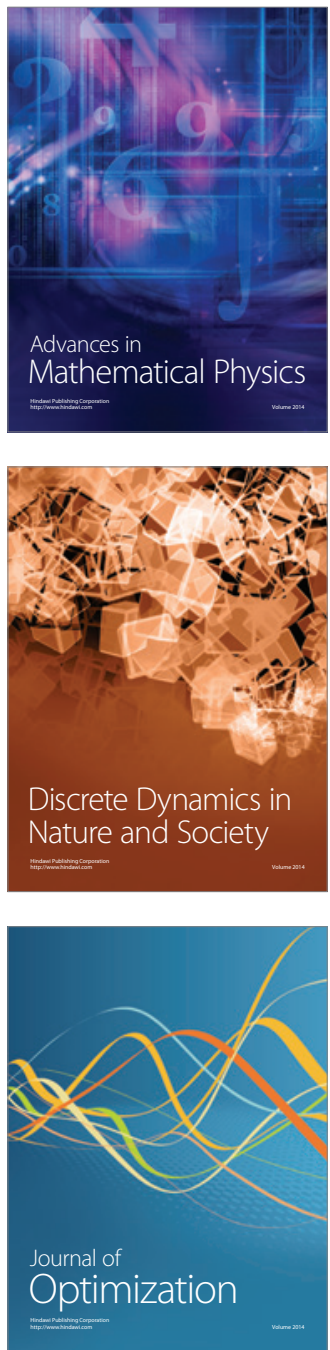